\title{
Influence of bulk doping type on the Li adsorption site on $\mathrm{Si}(111)-(1 \times 1): H$
}

\author{
J. J. Paggel, ${ }^{1, *}$ W. Mannstadt,,${ }^{2, \dagger}$ Chr. Weindel, ${ }^{2}$ M. Hasselblatt, ${ }^{3}$ K. Horn, ${ }^{3}$ and D. Fick ${ }^{2}$ \\ ${ }^{1}$ Freie Universität Berlin, Institut für Experimentalphysik, 14195 Berlin, Germany \\ ${ }^{2}$ Philipps-Universität, Fachbereich Physik und Zentrum für Materialwissenschaften, 35032 Marburg, Germany \\ ${ }^{3}$ Fritz-Haber-Institut der MPG, Department of Molecular Physics, 14195 Berlin, Germany
}

(Received 29 July 2003; published 15 January 2004)

\begin{abstract}
The model surface $\mathrm{Si}(111)-(1 \times 1): \mathrm{H}$ is used as a substrate for the adsorption of submonolayer amounts of Li. For $n$-doped substrates a peak right at the conduction band minimum is found in photoemission spectra. The peak is absent if the experiment is conducted on $p$-type substrates. Density functional theory calculations for different adsorption sites correlate this peak in the conduction band with $\mathrm{Li}$ adsorption in a $\mathrm{H} 3$ site of the $\mathrm{Si}(111)-(1 \times 1): \mathrm{H}$ surface. Experiment and theory show that the binding energy of the spectral feature is independent of the Li coverage. The absence of the structure for $p$-type substrates suggests a doping dependence of the adsorption site for $\mathrm{Li}$ on $\mathrm{Si}(111)-(1 \times 1): \mathrm{H}$.
\end{abstract}

DOI: 10.1103/PhysRevB.69.035310

\section{INTRODUCTION}

Silicon is a simple semiconductor; all the native Si surfaces are, however, heavily reconstructed and therefore possess a large number of electronic surface states. ${ }^{1}$ These surface states pin the Fermi level within the band gap, and their existence complicates the study of the pinning behavior of the Fermi level at these surfaces as a function of metal coverage. Schottky barrier formation has thus often been investigated through metal adsorption on the (110) surface of compound semiconductors, where the surface states are often located in, or very close to, the bulk bands (see, for example, the Introduction of Ref. 2). The hydrogen-terminated Si(111) surface, with its "unreconstructed" character and its semiconducting surface without electronic surface states in the electronic band gap, has attracted a number of researchers following the discovery of the wet chemical preparation method. ${ }^{3}$ This method allows the preparation of highly perfect surfaces with very low defect densities. In combination with the small unit cell of the surface reconstruction (for a semiconductor surface), this suggests its use as model system for semiconductor surfaces. The formation of metal-tosemiconductor contacts can thus be studied with $\mathrm{Si}$ as substrate without the complication of surface states in the band gap. One unexpected complication arises from the fact that the Si $2 p$ core level spectra from this surface display more components than expected from the geometric arrangement of the $\mathrm{Si}$ atoms in the unit cell. ${ }^{4}$ While several models have been proposed, ${ }^{4-6}$ no final conclusion has been drawn so far, as core level photoemission from this system appears to be complex. Several groups have addressed the electronic structure in the valence region. ${ }^{3,7-9}$ The basic idea here was that the simple geometric arrangement and the small number of inequivalent atoms in the surface unit cell render the chemical and electronic processes simple enough to obtain access to the details of the surface electronic structure for a prototypical semiconductor surface.

The $\mathrm{Si}(111)-(1 \times 1): \mathrm{H}$ surface is obviously not as simple as experimentalists had anticipated. Because of the role of the hydrogen in the adsorption process, metal adsorption on this surface is also far from being simple. One of the main questions regarding the chemistry in the adsorption process
PACS number(s): 79.60.Dp, 68.43.Fg

is the following: Does hydrogen remain adsorbed, or does it react with the metal atoms? ${ }^{10,12}$ Several groups have already addressed alkali metal adsorption on $\mathrm{Si}(111)-(1 \times 1)$ : H. de Renzi and co-workers ${ }^{11}$ used the unpinned Fermi level of the substrate to investigate its inhomogeneous pinning for very low potassium coverage, while Grupp and Taleb-Ibrahimi ${ }^{12}$ investigated the pinning behavior over a larger range of alkali metal coverage. They found a position of the Fermi level inside the conduction band for some coverages. This had been seen before for Cs adsorption on $\operatorname{InAs}(110)$ by Aristov and co-workers. ${ }^{2,13}$ Hakansson and Johansson ${ }^{14}$ deposited $\mathrm{K}$ on the As-terminated $\mathrm{Si}(111)-(1 \times 1)$ surface and found a $\mathrm{K}$-induced surface state located at the Fermi level. In momentum space this peak is found in a pocket around the $\bar{M}$ point of the surface Brillouin zone. They interpreted this peak as population of a surface resonance by electrons from the $\mathrm{K}$ adsorbate. Recently, Biagi et al. ${ }^{15}$ published a highresolution photoemission study for $\mathrm{K}$ and $\mathrm{Cs}$ adsorption on the hydrogen-terminated (111) surface of $n$-doped Si, where they investigated the dispersion of the peak, confirming its origin as electron transfer into the Si bulk conduction band minimum at the $M$ point. The nature of this peak is identified as due to electron confinement in the surface layer. Earlier, Biagi and co-workers ${ }^{16}$ discussed the two-dimensional electron gas due to $\mathrm{K}$ adsorption on the hydrogen-terminated Si(111) surface by electron energy loss spectroscopy. Similar "gap states" are also observed for various alkali metals on different III-V semiconductor surfaces. ${ }^{17}$ These gap states are, however, not located at the conduction band minimum.

This is the background on which we present experimental data as well as results of $a b$ initio density functional theory calculations with the full-potential linear augmented-planewave (FLAPW) method $^{18}$ to interpret the experimental results for $\mathrm{Li}$ adsorption on a $\mathrm{Si}(111)-(1 \times 1)$ :H surface (compare also to Ref. 19).

\section{EXPERIMENT}

The spectra shown here were taken at the BESSY I (Berliner Speicherringgesellschaft für Synchrotronstrahlung $\mathrm{mbH})$ monochromator PM-5 with a Scienta SES 200 electron spectrometer in the angle integrating mode. Li was 


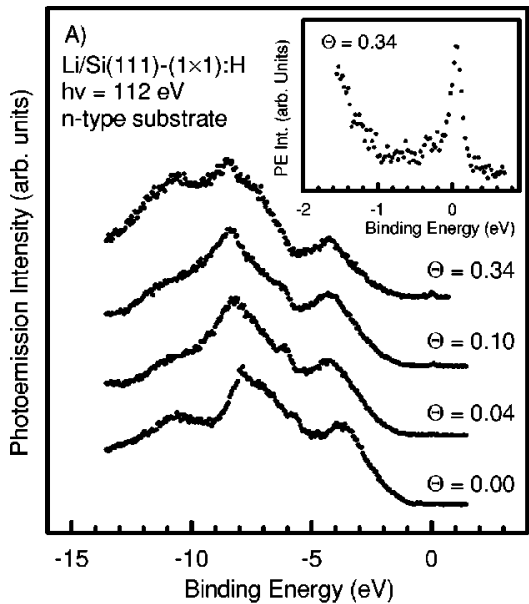

FIG. 1. (a) Valence band spectra for $\mathrm{Li}$ on $n$-doped $\mathrm{Si}(111)-(1 \times 1): \mathrm{H}$. Li coverage as inferred from the work function change is indicated on the individual traces. The inset shows the Liinduced narrow structure right at the Fermi level. (b) Similar Li coverages as in (a), but now with $p$-doped substrates. The previously pronounced structure is now barely visible (if present at all). The conduction band minimum is enlarged in the inset and shows a possible, very small structure about $0.6 \mathrm{eV}$ above the Fermi level. evaporated from commercial getter sources (SAES Getters SA) onto $n$-type (phosphorus) and $p$-type (boron) doped $\mathrm{Si}(111)$ wafers (Virginia Semiconductors) with a specific resistance of 5-10 $\Omega \mathrm{cm}$. The wafers were oriented within $0.5^{\circ}$ of the (111) direction. Sample preparation followed the published procedures for wet chemical preparation of $\mathrm{Si}(111)$ $(1 \times 1)$ : $\mathrm{H}$ surfaces. After insertion into the vacuum system, the samples were annealed briefly to remove volatile species from the surface, which initially pin the Fermi level. Sample quality and cleanliness were routinely checked using $\mathrm{x}$-ray photoemission survey spectra taken at $h \nu=850 \mathrm{eV}$, by inspection of the Si $2 p$ core level spectrum under surface sensitive conditions, and using the valence band signature. All three methods allow a reliable assessment of the sample quality. Experimental resolution of the spectrometer/ monochromator combination was set to about $60-120 \mathrm{meV}$, suitable for the experiments presented here. All spectra were taken at a sample temperature of about $100 \mathrm{~K}$, measured by a thermocouple located at the sample holder. Si $2 p$ core level spectra were taken at photon energies of about $112 \mathrm{eV}$ and $130 \mathrm{eV}$ in order to keep track of the position of the bulk valence band maximum and to assess the possibility of inhomogenous Fermi level pinning at the surface. Si $2 p$ spectra taken at $112 \mathrm{eV}$ photon energy are not extremely bulk sensitive and some surface information is left in the data. Extremely bulk sensitive spectra should show only a single spin-orbit pair. We will observe, however, an additional component. Lowering the photon energy even further will change the electron escape depth as a function of the Fermi level position in the band gap as the kinetic energy of the core level photoelectrons changes. The relative change in kinetic energy is smaller for a slightly larger kinetic energy. The photon energy used here is a safe compromise, yielding similar surface sensitivity at the same photon energy for both doping types. The valence band spectra were recorded at 112 $\mathrm{eV}$ photon energy. Using high photon energy reduces matrix element variations and the angle-integrated spectra represent the density of states in the valence band more closely.

\section{RESULTS AND DISCUSSION}

Figure 1 shows a comparison of valence band spectra for increasing coverage of $\mathrm{Li}$ on the $\mathrm{Si}(111)-(1 \times 1)$ : $\mathrm{H}$ surface of $p$-type (right) and $n$-type (left) doped substrates. The bottom traces show spectra for the clean surfaces. Since the samples are prepared using the wet chemical preparation method, the quality of the $n$-type substrates is not as high as that of the $p$-doped samples. ${ }^{20}$ Both surface modifications show the surface state at about $5 \mathrm{eV}$ below the valence band maximum. ${ }^{4}$ The surface state from the $p$-doped surface is more pronounced and sharper than the surface state on the $n$-doped samples, indicating a higher amount of disorder and lower level of perfection for the latter surface. Both surfaces, however, display an unpinned Fermi level. The amount of disorder on the $n$-doped substrate is thus not high enough to disturb the surface electronically. Si $2 p$ features (to be discussed later) are well structured and sharp for both sets of samples, such that laterally inhomogeneous pinning of the Fermi level cannot explain the broader features in the valence band spectra of the $n$-doped substrates. ${ }^{11}$

Exposing the $n$-type and $p$-type substrates to a flux of $\mathrm{Li}$ atoms causes changes in the valence band spectra of both samples: the surface state at $5 \mathrm{eV}$ binding energy with respect to the valence band maximum is suppressed, and the spectra from both samples show a rigid shift to higher binding energies.

Since all spectra presented here are taken at a temperature of about $100 \mathrm{~K}$, surface photovoltage ${ }^{21}$ (SPV) in principle has to be considered. Both SPV and band banding lead to shifts of the spectra. In the present case, the balance between carrier mobility and electron-hole pair generation is such that below a critical $\mathrm{Li}$ coverage of about $\Theta=0.7-1.0$ monolayers (ML) no surface photovoltage is observed under the conditions used. (For details of the SPV and its dependence on experimental parameters, see Ref. 22.) At this critical coverage, a sudden jump of the spectral features and valence band emission from energies above the reference Fermi level of the metallic sample holder are observed. This clear onset of the SPV effect at higher Li coverage indicates that, for the low coverage to be discussed here, the Fermi level position in the electronic band gap is known.

Expanding the $y$-axis scale in Fig. 2, one sees that already for $1 / 25$ of a monolayer of $\mathrm{Li}$ on the $n$-doped surface a tiny structure at the Fermi level appears. This feature becomes more pronounced with increasing Li coverage and develops 


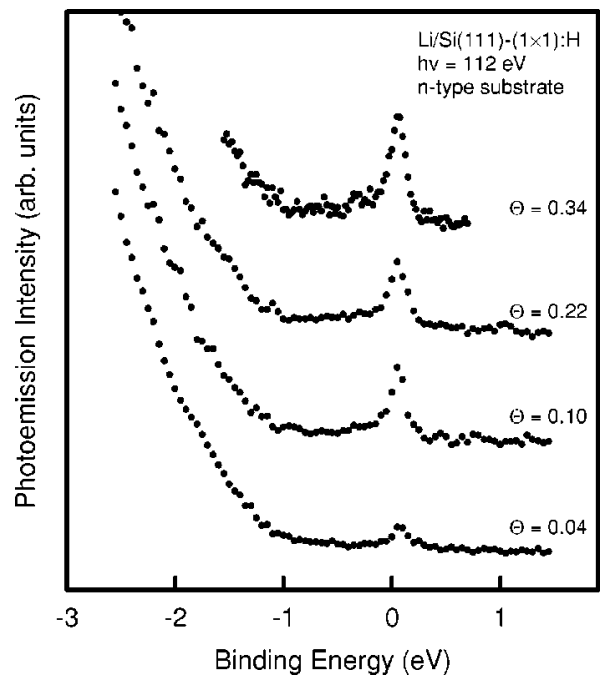

FIG. 2. Detailed view of the narrow peak structure at the Fermi level of the $n$-doped samples in the coverage range from 0.04 ML to 0.34 ML. The peak width and the peak position are hardly influenced by the Li coverage.

into a genuine peak in the photoemission spectrum as indicated in the inset of Fig. 1(a) and the upper traces in Fig. 2. The peak develops right at the bottom of the conduction band and is similar to what has been observed by other authors in different systems. ${ }^{2,13-15}$ The peak is smaller than in the spectra published in Ref. 15, since here we show angleintegrated data and the peak is localized in one specific area of reciprocal space.

Direct comparison with the spectra from the $p$-doped substrates gives an entirely different picture for the latter. The valence band maximum is close to the Fermi level for the clean surface, as it should be for an unpinned $p$-type surface, and moves to a midgap position for small $\mathrm{Li}$ coverage. There are no additional features in the fundamental band gap of the substrate with increasing Li coverage. The Fermi level position never approaches the conduction band minimum and always remains at a midgap position. In general, the Li film does not seem to inflict a change on the structure of the valence band of the substrates surface. A donation of a $\mathrm{Li}$ electron into a $\mathrm{Si}$ surface resonance or the bulk conduction band minimum as proposed by Biagi and co-workers ${ }^{15,16}$ is thus unlikely for the $\mathrm{Li}$ adsorption on the $p$-type substrate. The donation of electrons should work for both doping types, since the adsorption of a sizable fraction of a monolayer of $\mathrm{Li}$ overrules by far the bulk doping of the material in terms of carrier density. It should be noted that Biagi et al. used $n$-doped substrates only. Close inspection of the close-up of the valence band maximum [inset of Fig. 1(b)] reveals a tiny peak appearing $\sim 550 \mathrm{meV}$ above $E_{F}$. This peak is what is seen on the $n$-type surfaces, but the peak intensity is much smaller.

Figure 2 investigates the coverage dependence of the small peak at the Fermi level. A broadening of the peak is not observed, even if the Li coverage is tripled. An increasing carrier density in the proposed surface resonance should lead to an increased bandwidth, provided the filled band is not entirely flat and the instrumental broadening of the peak does
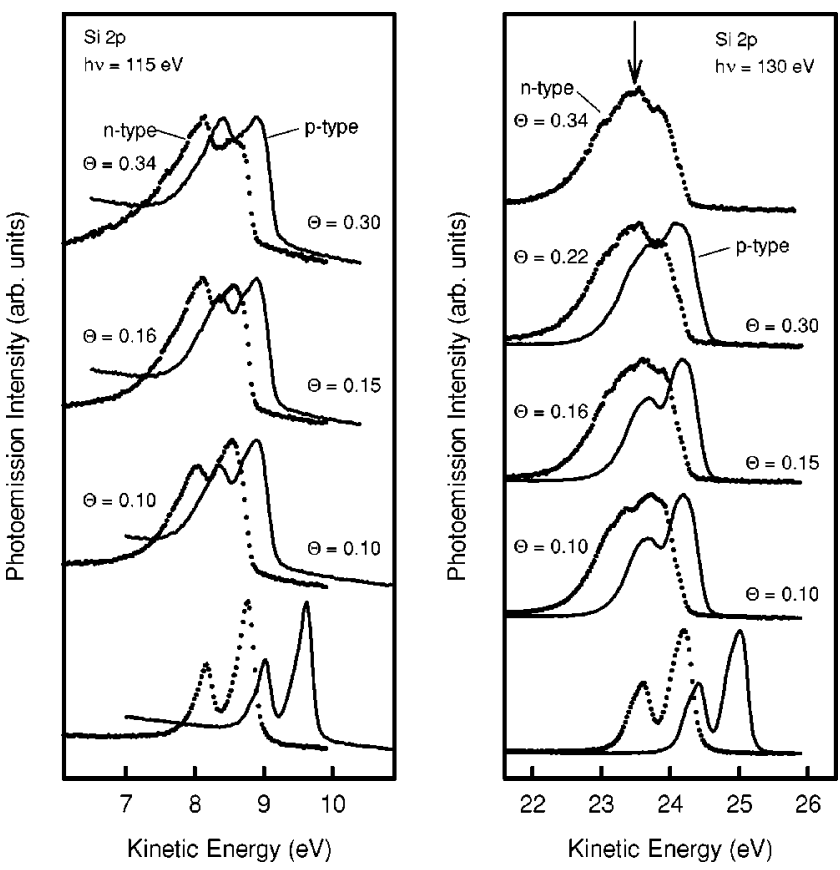

FIG. 3. Si $2 p$ core level spectra taken at about $112 \mathrm{eV}$ (left) and $130 \mathrm{eV}$ (right) photon energy. Dots denote data from $n$-doped samples, solid lines data from $p$-doped sample. Spectra from clean samples on the bottom, Li-covered samples stacked with Li coverage as indicated. The left panel shows mainly effects of the Fermi level position in the electronic band gap, while the right panel also gives information on the chemical reactions taking place at the surface.

not mask the effect. Since the angular acceptance of the spectrometer used and the photon energy employed guarantee the observation of the entire Brillouin zone in our experiments, the peak cannot be explained as filling of a surface resonance, and an alternative explanation for the peak is needed. This alternative explanation is given in Ref. 15 as population of a quantized state. In this way the width of the peak is indeed not limited by band filling, but by the splitting of subbands (minibands) in a quantum well structure. The quantum well nature of the peaks essentially leads to an unchanged peak width with Li coverage. (For details, refer to Ref. 15.)

For discussion of the pinning position of the Fermi level in the electronic band gap at the surface, it is much more appropriate to discuss core level spectra than the valence band emission. Figure 3 therefore shows bulk sensitive spectra taken at $112 \mathrm{eV}$ photon energy in the left panel, while the right panel shows surface sensitive spectra taken at $130 \mathrm{eV}$ photon energy. Spectra from both doping types are shown at similar Li coverage. The bottom traces show the spectra from the clean surfaces. The solid lines indicate the spectra from the $p$-doped samples. Dots mark the spectra from $n$-doped samples. The offset in the position of the spectra from the clean surfaces (bottom traces) just corresponds to the different positions of the Fermi level in the band gap of the unpinned surface. The difference in the binding energies for the Si $2 p$ core level in the two cases is $0.85 \mathrm{eV}$ and close to the expectation of roughly $1 \mathrm{eV}$ for the doping levels and sample 
temperature used here. ${ }^{23}$ Upon Li adsorption, for samples of both doping types, the Fermi level moves toward the bottom of the conduction band. For the $n$-type surface, the Fermi level seems to even move slightly into the conduction band itself (compare to Fig. 2) - just as previously reported in Refs. 12, 15, and 16. In the $p$-doped case, the Fermi level moves only to a midgap position. The difference of the two Fermi level positions with respect to the $\mathrm{Si}$ band edges is 325 $\mathrm{meV}$. For the coverage range investigated here, the Fermi level position is fixed after the deposition of sub-ML amounts of Li. For thicker films this will change (not shown here), but up to about 0.5 ML coverage the position of the Fermi level does not depend on the amount of Li deposited. The bulk sensitive spectra from $p$ - and $n$-doped samples look similar with respect to the line shape. Also, the Si $2 p$ core level lines are relatively narrow, demonstrating that the Fermi level pinning is rather homogenous at these surfaces. An inhomogenously pinned Fermi level increases the width of the core level spectra. Irrespective of other details that might differ between samples of the two doping types, the electrostatics in both cases is similar, and neither of the two sample types shows signs of an inhomogenous Fermi level position. The bulk sensitive core level spectra in the left panel of Fig. 3 change with Li coverage, since the photon energy used does not deliver extremely bulk sensitive data.

Considering the surface sensitive spectra in the right panel, the change of the pinning position is again evident, but now the line shape changes noticeably with Li coverage, and one observes a difference in the line shape for $n$-doped and $p$-doped samples. Since inhomogeous Fermi level pinning was ruled out by the bulk sensitive spectra, only chemical shifts can be responsible for the changed spectral line shapes. These chemical shifts are more pronounced in the case of the $n$-type sample than in the case of the $p$-type sample. The latter shows a well-separated spin-orbit pair until $0.15 \mathrm{ML} \mathrm{Li}$ coverage. The individual components increase in width, but no drastically shifted components are observed in the spectra. In the case of the $n$-type sample, a large chemical shift of about $300-400 \mathrm{meV}$ is found. This shifted component fills the valley between the $2 p_{3 / 2}$ and the $2 p_{1 / 2}$ components and actually produces a peak in the line shape in this location (arrow). After an initial change from the clean surface to a coverage of $\Theta=0.10 \mathrm{ML}$, the shape of the Si $2 p$ emission is again nearly unchanged for a coverage between $\Theta=0.16$ and $\Theta=0.34 \mathrm{ML}$. The line shapes of the core level spectra are not structured enough to allow a meaningful analysis in terms of surface core level shifts and/or chemical shifts, as the number of components needed to describe the data is neither known nor easily extractable from the spectra.

The difference in the appearance of the Si $2 p$ core level might be due to either the difference in doping type or the above mentioned difference in surface quality with increased disorder in the surface of the $n$-doped samples. The peak observed in the valence band of the $n$-type sample is the one also observed by Biagi et al. ${ }^{15}$ It is very narrow. Both facts render a possible origin from disorder in our samples unlikely, since (a) disorder leads to inhomogenously broadened peaks in spectra and (b) it is not likely that Biagi et al. have the exact kind of defects in their samples, generating the same peak in the valence band, that is also well behaved and allows analysis in terms of a quantum well structure (compare to Ref. 15). From the sample with a narrow valence band peak, we observe broader core level spectra. From the samples with better-defined core level spectra, we observe no peak in the valence band. If the correlation between core level and valence level spectra were reversed, disorder would be a good candidate for the explanation of the effect, i.e., in this case the less perfect sample with increased disorder would cause the small peak to disappear. In the present configuration, the narrow peak in the valence band indicates a rather low disorder with an apparently stronger chemical reaction observed at the Si $2 p$ core level.

The conclusion to be drawn from the surface sensitive core level spectra is thus that the chemical reactivity of the two different doping types toward $\mathrm{Li}$ adsorption is different. $p$-doped samples show smaller chemical shifts in the core level spectra than do $n$-doped samples.

Support for a meaningful interpretation of these results can be drawn from calculations using the framework of density functional theory (DFT). Only recently have such codes yielded reasonable band gaps for semiconductors without introducing additional adjustable parameters. The present calculations were performed with the P-FLAPW code, ${ }^{24}$ a massively parallel implementation of the FLAPW method. ${ }^{18}$ This specially designed code allows treating complex surface structures (as well as bulk systems) from first principles. The lower right panel of Fig. 4 shows the total density of states, with a well-developed band gap of $0.85 \mathrm{eV}$ (experimental value $^{25}$ of $1.153 \mathrm{eV}$ at $0 \mathrm{~K}$ ). The upper part of Fig. 4 shows the density of states for a $5 \times 5$ (right) and a $3 \times 3$ (left) supercell calculation of $\mathrm{Li}$ on the $\mathrm{Si}(111)-(1 \times 1): \mathrm{H}$ surface in a $\mathrm{H} 3$ adsorption site. Clearly, the development of Liinduced density of states at the Fermi level is seen, while the band gap of the semiconductor remains unaffected. Not shown are the projected results, which indicate the densities of states at $E_{F}$ to be entirely $s$-state $(l=0)$ dominated. Adsorbing $\mathrm{Li}$ instead in a substitutional site, replacing a hydrogen atom, produces a new structure in the valence band with a binding energy of about $250 \mathrm{meV}$ relative to the valence band maximum (lower left panel of Fig. 4) and puts the Fermi level in the center of the band gap.

Three features of calculation and experiment show excellent agreement. (a) Li induces a density of states at the bottom of the conduction band, which is actually occupied. (b) The density of states at the Fermi level is not influenced much by the coverage with the alkali metal. When going from $5 \times 5$ to $3 \times 3$, the coverage is increased by a factor of about 2.8. The density of states at the Fermi level is nearly unchanged. These two observations match the experimental results for the $n$-doped substrates, together with the position of the Fermi level right at or in the conduction band minimum. Looking into the details of the electron distribution provided by the DFT calculation, we find that the $\mathrm{Li}$ atom nearly completely loses its valence electron to the Si substrate, thus supporting the picture of doping the surface layer. The donated electrons are not located in the topmost layer, but in a backbond type configuration in the atomic plane just beneath the surface. ${ }^{26}$ 

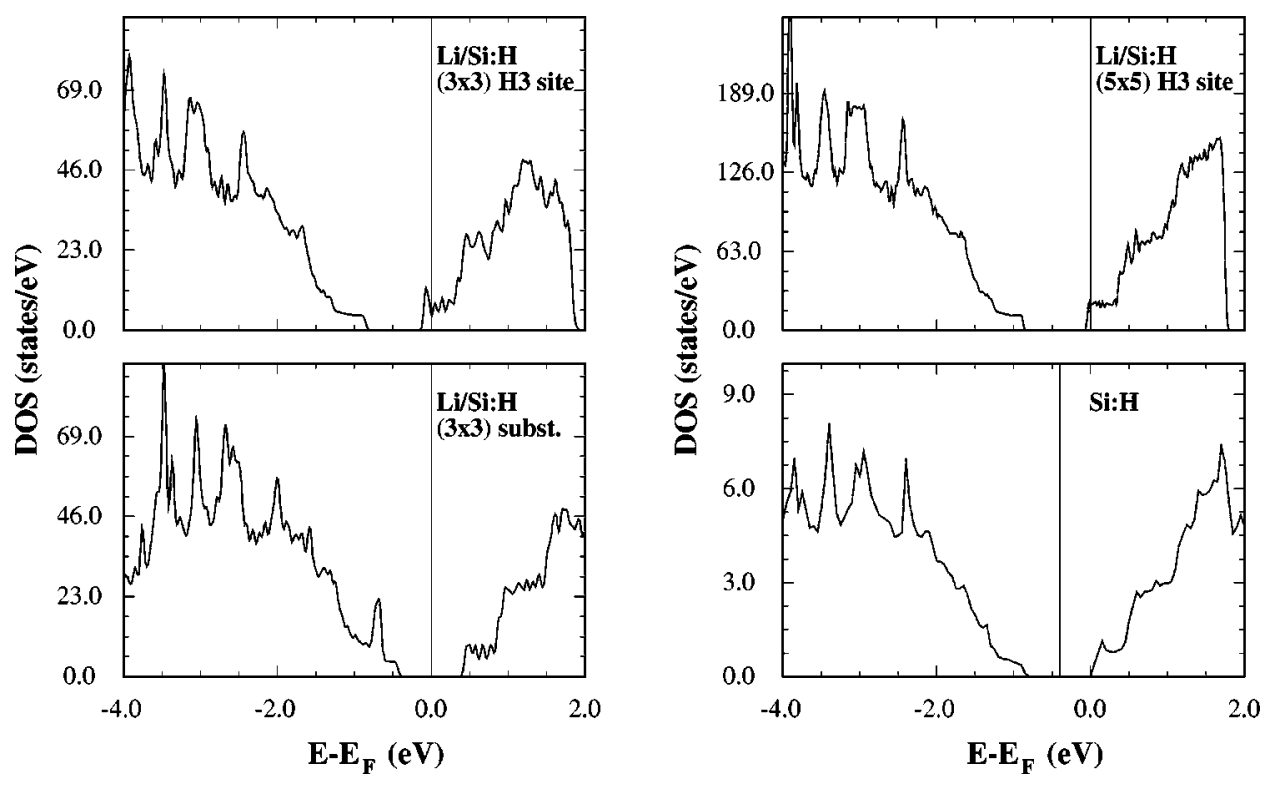

FIG. 4. Density of states obtained from a DFT calculation. Top left: $\mathrm{Li} / \mathrm{Si}(111)-(1 \times 1): \mathrm{H}$ in a relaxed $3 \times 3$ geometry. Top right: $5 \times 5$ geometry. Bottom left: $\mathrm{Li}$ in a $3 \times 3$ substitutional site. Bottom right: total density of states for the bare substrate.

The third feature that is similar between experiment and theory concerns the interpretation of the spectra from the $p$-doped samples, which so far do not seem to be reflected in the calculations. The calculation for the substitutional site puts the Fermi level in a midgap position. Also, no state density at the conduction band minimum is introduced. This agrees with the situation found in the experiment for the $p$-doped samples. Since the sample quality is higher for the $p$-doped than for the $n$-doped samples, defects as a reason for the missing structure can be safely excluded. On the other hand, defects are an unlikely cause of the structure observed for the $n$-doped case anyway, since the H3 adsorption site calculation reproduces the experimental result. The computation for the substitutional site predicts a narrow peak in the valence band for a binding energy of $\sim 0.3 \mathrm{eV}$ below the valence band maximum. This is not observed in the experiment. Possibly the real adsorption site is not a simple substitutional site.

By comparison of the experimental results to the theoretical calculation, we therefore propose a doping dependence of the adsorption site for $\mathrm{Li}$ adsorption on the $\mathrm{Si}(111)-(1$ $\times 1)$ : $\mathrm{H}$ surface. In the $n$-doped case $\mathrm{Li}$ atoms of the first fraction of a ML adsorb in the H3 adsorption site, while in the $p$-type case the first $\mathrm{Li}$ atoms adsorb in a differentpossibly substitutional-adsorption site. Doping dependence of the adsorption site is an unusual concept in surface physics and to our knowledge has not been observed before. The calculation itself is not doping dependent, i.e., the slab in the DFT calculation is electrically neutral and the unit cell is too small to include dopants for a reasonable doping concentration. An investigation for the doping type dependence of the adsorption site on the basis of the density functional theory, albeit highly desirable, can thus not be given. Since the doping type influences the electrochemical potential at the surface, one in fact could expect such behavior at a semiconductor surface without electronic surface states in the electronic band gap. The electrochemical potential differs by about $1 \mathrm{eV}$ at the unpinned $\mathrm{Si}$ surface between $n$ - and $p$-type materials. Adding one more electron to this system will thus change the total electronic energy by a different amount. Differences in chemical reactivity for different doping types and even doping levels are well known in the area of microfabrication, where they are efficiently used as etch stops. (Compare, for example, to Ref. 27.)

This electronic difference might well tip the energy balance between two electronically inequivalent but energetically similar adsorption sites. This difference in the electronic structure of the adsorption site after the adsorption of the Li atom can also be inferred from the different core level shifts observed in surface sensitive spectra from the Si $2 p$ core level. It should be mentioned that up to now no detailed quest for the optimized adsorption site of $\mathrm{Li}$ on $\mathrm{Si}(111)$-(1 $\times 1)$ : $\mathrm{H}$ has been performed. For the two sites tried so far, the geometries were optimized with respect to the total energy of the system.

In order to obtain more information about the local properties of adsorbed $\mathrm{Li}$ on this surface, $\beta$-NMR experiments were performed on $\mathrm{Li}$ adsorbed at a coverage of about 0.01 ML and 0.25 ML on the Si(111)- $(1 \times 1)$ :H surface. Surprisingly enough, no difference in $T_{1}$ times was observed. ${ }^{28}$

\section{CONCLUSIONS}

In summary, the adsorption of $\mathrm{Li}$ on the model semiconductor surface $\mathrm{Si}(111)-(1 \times 1): \mathrm{H}$ has been investigated using photoelectron spectroscopy and DFT calculations. We find a marked difference in Fermi level pinning for this system between $p$ - and $n$-type substrates and observe an occupied surface state near the conduction band minimum only on $n$-type substrates. This suggests that the adsorption site of Li on this model surface is strongly influenced by the type of doping of the silicon substrate. For the $n$-doped substrates, good agreement between calculated electronic structure and our valence band data was found for the $\mathrm{H} 3$ adsorption site. The difference in the observed photoemission spectra is explained by a 
bulk doping type dependence of the adsorption site of the $\mathrm{Li}$ atoms on the $\mathrm{Si}(111)-(1 \times 1): \mathrm{H}$ surface. A doping dependence of the Si $2 p$ core level line shape-which is generally a good indicator for chemical reactivity and especially differences therein-is observed. Such behavior has to our knowledge not been reported before. The influence of bulk doping on the chemical reactivity is well known in semiconductor processing. An influence of the bulk doping type (and

*Corresponding author. FAX: +49 (30) 838 56299. Email address: Jens.Paggel@physik.fu-berlin.de

†Present address: Schott Glas, Service Division Research and Development, 55014 Mainz, Germany.

${ }^{1}$ D. Haneman, Rep. Prog. Phys. 50, 1045 (1987).

${ }^{2}$ V. Yu Aristov, G. LeLay, P. Soukiassian, K. Hricovini, J.E. Bonnet, J. Osvald, and O. Olsson, J. Vac. Sci. Technol. B 12, 2709 (1994).

${ }^{3}$ P. Dumas, Y.J. Chabal, and G.S. Higashi, Phys. Rev. Lett. 65, 1124 (1990).

${ }^{4}$ K. Hricovini, R. Günther, P. Thiry, A. Taleb-Ibrahimi, G. Indlekofer, J.E. Bonnet, P. Dumas, Y. Petroff, X. Blasé, X. Zue, S.G. Louie, Y.J. Chabal, and P.A. Thiry, Phys. Rev. Lett. 70, 1992 (1993).

${ }^{5}$ C.K. Karlsson, F. Owman, E. Landemark, Y.-C. Chao, P. Mårtensson, and R.I.G. Uhrberg, Phys. Rev. Lett. 72, 4145 (1994).

${ }^{6}$ X. Blasé, X. Zhu, and S.G. Louie, Phys. Rev. B 49, 4973 (1994).

${ }^{7}$ Y. He, S. Bouzidi, B.-Y. Han, L.-M. Yu, P.A. Thiry, R. Caudano, and J.-M. Debever, Phys. Rev. B 54, 17654 (1996).

${ }^{8}$ S. Bouzidi, F. Coletti, J.-M. Debever, P.A. Thiry, P. Dumas, and Y.J. Chabal, Phys. Rev. B 45, 1187 (1992).

${ }^{9}$ S. Gallego, J. Avila, M. Martin, X. Blase, A. Taleb, P. Dumas, and M.C. Asensio, Phys. Rev. B 61, 12628 (2000).

${ }^{10}$ K. Fukutani, H. Iwai, Y. Murata, and H. Yamashita, Phys. Rev. B 59, 13020 (1999).

${ }^{11}$ V. de Renzi, R. Biagi, U. del Pennino, M. Pedio, A. Goldoni, and R. Larciprete, Phys. Rev. B 62, R10 657 (2000).

${ }^{12}$ C. Grupp and A. Taleb-Ibrahimi, Surf. Sci. 408, 160 (1998).

${ }^{13}$ V. Yu Aristov, G. LeLay, V.M. Zhilin, G. Indlekofer, C. Grupp, A. Taleb-Ibrahimi, and P. Soukiassian, Phys. Rev. B 60, 7752 (1999).

${ }^{14}$ M.C. Håkansson and L.S.O. Johansson, Surf. Sci. 342, 293 (1995). maybe also doping level) on the adsorption site is thus not totally unexpected and might also be present in other cases.

\section{ACKNOWLEDGMENTS}

We acknowledge support by the Bundesministerium für Bildung und Forschung through the Verbundprojekt 22. We thank the John-von-Neumann Institute for Computing in Jülich (Germany) for computer time on the Cray T3E.

${ }^{15}$ R. Biagi, P. Fantani, V. De Renzi, M.G. Betti, C. Mariani, and U. del Pennino, Phys. Rev. B 67, 155325 (2003).

${ }^{16}$ R. Biagi, L. Rettighieri, U. del Pennino, V. Panella, and P. Dumas, Surf. Sci. 402, 547 (1998).

${ }^{17}$ D.A. Evans, G.J. Lapeyre, and K. Horn, Phys. Rev. B 48, 1939 (1993).

${ }^{18}$ E. Wimmer, H. Krakauer, M. Weinert, and A.J. Freeman, Phys. Rev. B 24, 864 (1981).

${ }^{19}$ H. Winnefeld, M. Czanta, G. Fahsold, H.J. Jänsch, G. Kirchner, W. Mannstadt, J.J. Paggel, R. Platzer, R. Schillinger, R. Veith, C. Weindel, and D. Fick, Phys. Rev. B 65, 195319 (2002).

${ }^{20}$ The etching process itself needs the presence of holes in the valence band. These holes are already present in $p$-doped samples. For $n$-type doping, they have to be generated by illumination of the sample [see H. Gerischer, P. Allongue, and V. Costa-Kieling, Ber. Bunsenges. Phys. Chem. 97, 753 (1993)]. In our experience, it is in general easier to prepare $p$-type samples than to prepare $n$-type samples.

${ }^{21}$ M. Alonso, R. Cimino, and K. Horn, Phys. Rev. Lett. 64, 1947 (1990).

${ }^{22}$ M.H. Hecht, Phys. Rev. B 41, 7918 (1990).

${ }^{23}$ S.M. Sze, Physics of Semiconductor Devices, 2nd ed. (Wiley Interscience, New York, 1981).

${ }^{24}$ A. Canning, W. Mannstadt, and A.J. Freeman, Comput. Phys. Commun. 130, 233 (2000).

${ }^{25}$ CRC Handbook of Physics and Chemistry, edited by David R. Lide (Chemical Rubber Company, Boca Raton, FL, 2003).

${ }^{26}$ W. Mannstadt (unpublished).

${ }^{27}$ Marc J. Madou, Fundamentals of Microfabrication: The Science of Miniaturization (CRC Press, Boca Raton, FL, 2002).

${ }^{28}$ C. Weindel, M. Czanta, H.J. Jänsch, G. Kirchner, J.J. Paggel, H. Winnefeld, and D. Fick (unpublished); C. Weindel, Ph.D. thesis, Philipps Universität, Marburg, 2001. 